\title{
Nanoscale
}

A) Check for updates

Cite this: Nanoscale, 2021, 13, 18148

\section{Dopamine based adhesive nano-coatings on extracellular matrix (ECM) based grafts for enhanced host-graft interfacing affinity $\dagger$}

\author{
Chao Tao, ${ }^{a, b}$ Min Jin, ${ }^{b}$ Hang Yao*c and Dong-An Wang (iD *a,b,d
}

Received 24th September 2021, Accepted 4th October 2021

DOI: 10.1039/d1nr06284k

rsc.li/nanoscale

\begin{abstract}
Interfacing affinity between grafts and host tissues is an urgent issue that needs to be addressed for the clinical translation of tissue engineered extracellular matrix (ECM) based grafts. Dopamine is known as a universal adhesive, the catechol groups on which could form chelating bonds with metal ions. Herein we developed an adhesive nano-coating on ECM based grafts which could crosslink in situ with ferric ions for fixation with surrounding tissues after implantation without affecting the porous structures of the grafts. Therefore, decellularized living hyaline cartilage graft (dLhCG), a model ECM-based graft, with dopamine based natural biological material adhesive coatings was manufactured to address the interfacing affinity issue between ECM-based grafts and cartilage. A macromolecule backbone was needed for the coating material to avoid the formation of a rigid crosslinking system and adverse effects caused by small molecules of dopamine. Chondroitin sulfate (CS), a cartilage derived sulfated GAG, was chosen as the backbone to fabricate dopamine modified CS (CSD) with no impurities introduced to the joint. Dopamine modified serum albumin (BCD) was also chosen for the favorable biocompatibility of albumin. Both dLhCG coated with CSD and dLhCG coated with BCD showed enhanced adhesive strength with cartilage after chelating with ferric ions in situ compared to dLhCG and further potential in improving the interfacing affinity of dLhCG with cartilage.
\end{abstract}

\section{Introduction}

Integration or fixation of grafts or devices to tissues is a big challenge for successful implantation from a clinical perspective. ${ }^{1,2}$ For example, it is reported that dislocation of implanted mesh in a preperitoneal mesh hernia repair would remarkably increase the recurrence rate. ${ }^{3}$ Furthermore, incomplete immobilization in transplantation will result in graft failure. ${ }^{4}$ Extracellular matrix (ECM)-derived xenografts are proved to be desirable tissue engineering scaffolds with a porous structure, a hospitable microenvironment for cells to attach and proliferate with abundant donating sources. For example, our group has developed an ECM based tissue engineered xenograft called decellularized living hyaline cartilage

\footnotetext{
${ }^{a}$ Karolinska Institutet Ming Wai Lau Centre for Reparative Medicine, HKSTP, Sha Tin, Hong Kong SAR. E-mail: dwang229@cityu.edu.hk

${ }^{b}$ Department of Biomedical Engineering, City University of Hong Kong, 83 Tat Chee Avenue, Kowloon, Hong Kong SAR

${ }^{c}$ School of Chemistry and Chemical Engineering, Yangzhou University, Yangzhou 225009, Jiangsu, P. R. China. E-mail: yaohang@yzu.edu.cn

${ }^{d}$ Shenzhen Research Institute, City University of Hong Kong, Shenzhen, P. R. China $\dagger$ Electronic supplementary information (ESI) available. See DOI: 10.1039/ d1nr06284k
}

graft (dLhCG). ${ }^{5-11}$ Unlike wound closure, it is crucial to preserve the porous structures of implanted grafts when adhesives for fixation of grafts are applied. Therefore, the current commercially-available and commonly used fibrin glue, TISSEEL, with a mechanism inspired by clotting with two components, thrombin and fibrinogen, ${ }^{12}$ might not be sufficient for the fixation of ECM based grafts. Various kinds of absorbable or non-absorbable sutures, ${ }^{13-18}$ staples ${ }^{19}$ and adhesive tapes were available for fixation of grafts or medical devices such as skin grafts, vascular grafts and cardiac grafts. However, sutures and staples are limited in damaging surrounding tissues, eliciting inflammatory responses, scar tissue formation and possible anesthetics administration. ${ }^{20}$ Moreover, sutures and staples are not applicable for porous, sponge-like ECM based grafts due to the damage caused to the structure and challenge of the surgeon skills. Adhesive tapes are used in limited application in wet and hairy areas. ${ }^{21}$ Therefore, an adhesive to enhance the adhesion between grafts and tissues with an interpenetrating crosslinking structure by chemical reactions or physical interactions with good biocompatibility and less impurities needs to be developed.

Existing biomaterials studied for grafts' or devices' adhesives or glues ${ }^{22-30}$ covered proteins such as fibrin, ${ }^{25}$ gelatin, ${ }^{31,32}$ and albumin, ${ }^{33}$ polysaccharides $^{34,35}$ such as 
dextran and chitosan and synthetic polymers such as poly (ethylene glycol) (PEG) and ${ }^{36-38}$ polyurethanes (PU), ${ }^{39}$ which are biocompatible with sufficient mechanical strength. Moreover, adhesives should be capable of adhering to surrounding tissues by chemical bonds, interpenetration or physical linkage such as hydrogen bonds. ${ }^{20}$ Binding of adhesives to tissues could be ascribed to covalent bonds formed, for example, between cyanoacrylate adhesives ${ }^{40}$ or aldehydes on $\operatorname{dextran}^{41}$ and amines on the lysine of tissue proteins, and adhesion could also be achieved by an interpenetrating network formed between the polymerized or crosslinked adhesives and extracellular proteins in the tissue. ${ }^{42}$ Synthetic polymers or functionalized natural polymers with reactive groups could polymerize or crosslink in situ initiated by photo, heat, hydrolysis or crosslinkers and generate an interpenetrating network with tissue proteins, achieving adherence with surrounding tissues. However, irradiation is usually needed for polymerization and crosslinking, resulting in limitation in applicable areas in the bodies, and the free radicals produced in the polymerization would be harmful to healthy tissues.

Bio-inspired adhesives including mussel-inspired, geckoinspired ${ }^{43}$ and endoparasitic worm $-{ }^{44}$ inspired adhesives have been paid attention in recent years. ${ }^{20}$ The association of catechol and amine was believed to contribute to the adhesion of mussels, which inspired the synthesis of various kinds of catecholamines. Mussel-inspired adhesives have drawn more and more attention due to their biocompatibility, strong and nonselective adhesion to various surfaces. Among mussel-inspired catecholamines, dopamine was widely used due to its low cost and simple structure. Although not well established, according to existing theories, the adhesion and cohesion of dopamine originated from self-polymerization/oxidation to polydopamine in a mild alkaline environment initiated by oxygen. ${ }^{23}$ The adhesion was widely believed to be resulted from $\pi-\pi$ stacking, van der Waals forces and hydrogen bonding. ${ }^{45}$ Moreover, it is reported that chelating bonds between the catechol groups on polydopamine and metals or metal oxides were formed and would strengthen the adhesion. ${ }^{45}$

Moreover, an additional procedure of gluing grafts to surrounding tissues needs to be conducted during the surgery, which increased the technical complexity and operation time. We thereby considered introducing adhesive tissue-engineered grafts to have the grafts fixed in the defects in situ by simply adding ferric ions.

Polydopamine is a promising candidate as a tissue adhesive material. Our group has developed two kinds of dopaminefunctionalized tissue bio-adhesives with bovine serum albumin (BSA) ${ }^{33}$ and chondroitin sulfate $(\mathrm{CS})^{46}$ as backbones, respectively. The oxidation of dopamine in a mild basic environment and chelating bonds between catechol groups and ferric ions endowed them with adhesive properties. Tissue bio-adhesives showed sound seroma prevention and liver hemorrhage repair with excellent biocompatibility and potential in diverse surgical applications such as incision closure. However, polydopamine based adhesives were mainly investigated for wound closure currently. Due to diverse interactions derived from the catechol groups, we introduced and designed adhesive ECM-based grafts with nano-coatings, in which adhesion was based on the oxidation/polymerization of catechol groups and cohesion was based on chelation with ferric ions. Thereby, the integration of grafts with surrounding tissues can be achieved by simply adding ferric ion solution during the surgery. The surgery process can be simplified by combining bio-adhesives and grafts pre-surgery. Moreover, adhesive coatings are nanoscale in size and will not affect the porous structures of the grafts. Cells will migrate, proliferate and develop in the grafts post-implantation. Therefore, after a period of time post-implantation, the grafts will finally integrate with surrounding tissues through the ECM secreted by migrated cells and help in tissue regeneration. In summary, the adhesive nano-coatings work as bio-adhesives during the surgery and in the early stage of regeneration to enhance the interfacing affinity between grafts and surrounding tissues.

In this study, two kinds of adhesive prepolymer based on dopamine with CS and SA as the backbone were coated on dLhCG and chelated with ferric ions in situ for the enhancement of the interfacing affinity of dLhCG in cartilage defects without affecting the porous structure of dLhCG. The basic idea illustrating this technique is shown in Fig. 1. A polymer backbone was needed to eliminate the small molecules of dopamine which may be detrimental to human and avoid the formation of a rigid crosslinking structure. CS, a cartilage derived sulfate glycosaminoglycan (GAG), was used as the main chain with no impurities introduced. Moreover, another kind of natural biological material SA, a commonly used biocompatible protein, was also used as the backbone. The adhesive dLhCG was evaluated from aspects including morphology, adhesion strength and cytotoxicity.

\section{Materials and methods}

\subsection{Materials}

All the chemicals were purchased from Sigma Aldrich unless otherwise mentioned. $1 \times$ phosphate buffered saline (PBS) was purchased from Gibco. Tris- $\mathrm{HCl}$ buffer $(1.0 \mathrm{M}, \mathrm{pH}=8.8)$ was purchased from 1st Base Pte Ltd, Singapore.

\subsection{Preparation of dopamine modified chondroitin sulfate (CSD) and dopamine modified albumin (BCD)}

Dopamine modified chondroitin sulfate (CSD) was prepared based on an N-(3-dimethylaminopropyl)-N'-ethylcarbodiimide hydrochloride/ $N$-hydroxysuccinimide (EDC/NHS) coupling reaction. ${ }^{46}$ First, $0.5 \mathrm{~g}$ of chondroitin sulfate sodium salt (CS) was dissolved in $1 \times$ PBS and transferred to a two-neck flask. Then EDC (0.17 g) and NHS (0.12 g) were added and the carboxyl groups were activated for $1 \mathrm{~h}$ under $\mathrm{N}_{2}$ protection at $37{ }^{\circ} \mathrm{C}$ with $\mathrm{pH}$ 5.5. $0.23 \mathrm{~g}$ of dopamine hydrochloride in $1 \times$ PBS was subsequently added followed by another $1 \mathrm{~h}$ of $\mathrm{N}_{2}$ bubbling to remove oxygen in the system and avoid the oxidation of dopamine during the reaction. The mixture reacted under $\mathrm{N}_{2}$ protection at $37^{\circ} \mathrm{C}$ with $\mathrm{pH} 5.5$ for $6 \mathrm{~h}$. The final 


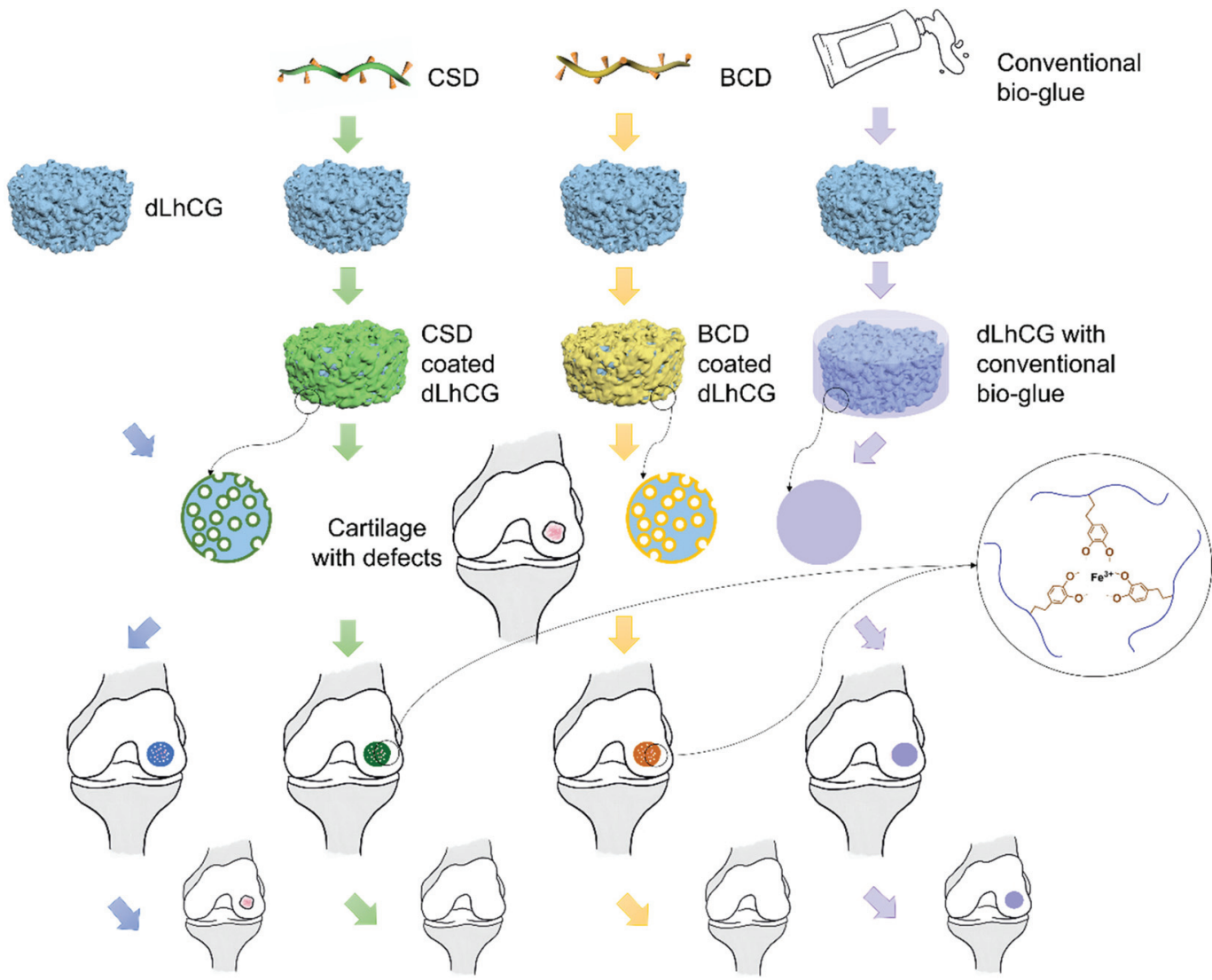

Fig. 1 Schematic illustration of the technique design.

product CSD was collected and dialyzed against deionized (DI) water for 2 days followed by freeze-drying (BT48, Millrock Technology Inc, USA).

Dopamine modified albumin (BCD) was also prepared based on an EDC/NHS coupling reaction. ${ }^{33}$ First, $0.5 \mathrm{~g}$ of citric acid (CA) was dissolved in $1 \times$ PBS and added to a one-neck flask followed by the addition of $0.4 \mathrm{~g}$ of EDC and $0.3 \mathrm{~g}$ of NHS. $1.85 \mathrm{~g}$ of bovine serum albumin (BSA) in $10 \mathrm{of} \mathrm{mL} 1 \times$ PBS was added dropwise afterwards. The mixture reacted under $\mathrm{N}_{2}$ protection at $37^{\circ} \mathrm{C}$ with $\mathrm{pH} 5.5$ for $6 \mathrm{~h}$. The prepolymer BCA was obtained after dialysis and freeze-drying of the reaction mixture. Subsequently, BCA $(0.75 \mathrm{~g})$ was dissolved in $1 \times$ PBS and transferred to a two-neck flask with an $\mathrm{N}_{2}$ inlet. EDC $(0.52 \mathrm{~g})$ and NHS $(0.38 \mathrm{~g})$ were added, after which the carboxyl groups were activated for $1 \mathrm{~h}$ with $\mathrm{pH}$ 5.5. Then dopamine hydrochloride $(0.63 \mathrm{~g})$ was dissolved in $1 \times$ PBS and added to the mixture after $1 \mathrm{~h}$ of $\mathrm{N}_{2}$ bubbling to remove oxygen in the system and avoid the oxidation of dopamine during the reaction. The mixture reacted under $\mathrm{N}_{2}$ protection at $37^{\circ} \mathrm{C}$ with $\mathrm{pH}$ 5.5. The final product $\mathrm{BCD}$ was obtained after dialysis against DI water for 2 days and lyophilization.

Chemical structures of the products mentioned above were characterized by attenuated total reflection-Fourier transform infrared (ATR-FTIR) spectroscopy (PerkinElmer Spectrum One) and proton nuclear magnetic resonance $\left({ }^{1} \mathrm{H}\right.$ NMR) spec- troscopy (Bruker Avance II $300 \mathrm{MHz}$ NMR) with deuterium oxide $\left(\mathrm{D}_{2} \mathrm{O}\right)$ as the solvent.

\subsection{Fabrication of decellularized living hyaline cartilage graft} (dLhCG)

dLhCG was fabricated according to our previous publications. ${ }^{9-11}$ Porcine chondrocytes were collected from porcine cartilage and embedded in alginate hydrogel together with gelatin microspheres. Chondrocytes grew and proliferated in the 3D hydrogel, secreting the ECM for graft establishment. Removal of alginate hydrogels was carried out using sodium citrate solution. Afterwards, LhCG was collected and decellularized by physical, chemical and enzymatic methods for the fabrication of dLhCG.

\subsection{Fabrication of CSD or BCD coated dLhCG and chelation with $\mathrm{Fe}^{3+}$ ions}

dLhCG was immersed in CSD $\left(200 \mathrm{mg} \mathrm{mL}^{-1}\right)$ or BCD $(50 \mathrm{mg}$ $\left.\mathrm{mL}^{-1}\right)$ solution in Tris- $\mathrm{HCl}$ buffer $(10 \mathrm{mM}, \mathrm{pH} 8.5)$ at room temperature (RT) for $1 \mathrm{~h}$, one group of which was immersed in $150 \mu \mathrm{L}$ solution without washing (C/B-dLhCG-no wash), while the other group was immersed in $500 \mu \mathrm{L}$ solution and washed three times with DI water after coating (C/B-dLhCG-wash). The weights of dLhCG both before and after coating were recorded 
to calculate the mass of CSD or BCD. CSD or BCD coated dLhCG was freeze-dried and kept at $4{ }^{\circ} \mathrm{C}$ for further use.

C/B-dLhCG-wash or C/B-dLhCG-no wash swelled in Tris$\mathrm{HCl}$ buffer (10 mM, pH 8.5) first, after which $\mathrm{FeCl}_{3}$ of certain concentration was added for chelation of CSD/BCD with $\mathrm{Fe}^{3+}$ and chelated C/B-dLhCG-wash (CFe/BFe-dLhCG-wash) or C/B-dLhCG-no wash (CFe/BFe-dLhCG-no wash) was prepared.

\subsection{Dynamic light scattering (DLS)}

DLS was conducted for the measurement of the size distribution of CSD or BCD in aqueous solution. The size distribution of BSA and CS was also measured as a control. The samples were dissolved in DI water before measurement.

\subsection{Atomic force microscope (AFM)}

The surface nanostructure of $\mathrm{CSD}$ or BCD coating was observed using an AFM (Agilent 5500 AFM, USA) in contact mode. CSD or BCD was dissolved in Tris-HCl buffer $(10 \mathrm{mM}$, $\mathrm{pH}$ 8.5), coated on a mica surface and dried at RT overnight before observation.

\subsection{Water contact angle}

CSD or BCD was dissolved in Tris-HCl buffer (10 mM, pH 8.5), coated on a porcine cartilage surface and dried at room temperature for $2 \mathrm{~h}$. The water contact angles of CSD or BCD coated porcine cartilage surfaces and uncoated surface were measured on a Kruss DSA25 Contact Angle Analyzer with a $5 \mu \mathrm{L}$ DI water drop for evaluating the feasibility of CSD or BCD to form a coating on dLhCG.

\subsection{Morphology of grafts}

The morphology of grafts was observed by scanning electron microscopy (SEM, JEOL JSM 6700F, Japan). All samples were freeze-dried and coated with platinum by sputtering (JFC-1600, JEOL Asia Pte. Ltd, Japan) for $150 \mathrm{~s}$ at $10 \mathrm{~mA}$ before observation.

\subsection{Lap shear strength test}

First of all, the adhesion of two slices of cartilage with CSD or BCD as adhesives was measured for comparison and for optimization of the CSD or BCD concentration, $\mathrm{Fe}^{3+}$ concentration and incubation time. CSD or BCD in Tris-HCl buffer of certain concentration $\left(500 \mathrm{mg} \mathrm{mL}{ }^{-1}, 200 \mathrm{mg} \mathrm{mL}^{-1}, 100 \mathrm{mg}\right.$ $\mathrm{mL}^{-1}$ and $50 \mathrm{mg} \mathrm{mL}^{-1}$ ) was mixed with $\mathrm{FeCl}_{3}$ Tris-HCl buffer solution of certain concentration $(200 \mathrm{mM}, 100 \mathrm{mM}, 50 \mathrm{mM}$ and $0 \mathrm{mM}$ ) in situ on one slice of cartilage, after which the two slices of cartilage were glued together. Subsequently, the adhesive was dried in situ with a hair dryer for $20 \mathrm{~min}$. Then the samples were kept at $37{ }^{\circ} \mathrm{C}$ under a high humid atmosphere by incubation in a water bath, but not immersed in water for $15 \mathrm{~min}, 1 \mathrm{~h}$ and $3 \mathrm{~h}$, respectively. ${ }^{23,33,46-48}$

The adhesion between CFe-dLhCG or BFe-dLhCG and cartilage was measured by a lap shear strength test. First, two slices of cartilage with a dimension of around $2 \mathrm{~mm} \times 8 \mathrm{~mm} \times$ $20 \mathrm{~mm}$ were collected from commercially available porcine bones. The exposed surface of cartilage was shaved with a knife to mimic the cartilage surface of osteoarthritis patients and used for adhesion. A hole with a diameter of $6 \mathrm{~mm}$ was punched on one slice of cartilage and filled with dLhCG or dLhCG with adhesive nano-coatings. C/B-dLhCG was swelled with Tris- $\mathrm{HCl}$ buffer (10 mM, pH 8.5) in situ first followed by the addition of $\mathrm{FeCl}_{3}$ in Tris-HCl buffer. Then the other slice of complete cartilage was adhered to the one embedded with CFe/BFe-dLhCG. Afterwards, the adhesive was dried in situ with a hair dryer for $20 \mathrm{~min}$. Then the samples were incubated at $37{ }^{\circ} \mathrm{C}$ in a water bath without immersion in water for $3 \mathrm{~h}$. C/B-dLhCG and dLhCG infiltrated with only Tris-HCl buffer or $\mathrm{FeCl}_{3}$ in Tris-HCl buffer were also tested as controls.

The lap shear strength test was conducted on a tensile meter (5543 Tensile Meter) and is shown in Fig. 7a.

\subsection{In vitro cytotoxicity}

In vitro cytotoxicity of CFe-dLhCG and BFe-dLhCG on chondrocytes was evaluated using a cell proliferation reagent WST-1 assay kit (Sigma Aldrich, Singapore). First, chondrocytes were cultured in 96 well plates (2500 cells per well) and incubated at $37{ }^{\circ} \mathrm{C}$ in an incubator in contact with CFe-dLhCG and BFedLhCG for $1 \mathrm{~d}, 4 \mathrm{~d}$ and $7 \mathrm{~d}$, respectively. $10 \mu \mathrm{L}$ of WST-1 was added to a $100 \mu \mathrm{L}$ culture medium at the corresponding time points and incubated together with chondrocytes for $3 \mathrm{~h}$ at $37{ }^{\circ} \mathrm{C}$ in $\mathrm{a}^{\mathrm{CO}_{2}}$ incubator. The absorbance of the incubated mixture was measured and calculated on a microplate reader (Thermo Electron Corporation, USA) based on the manufacturer's protocol. The cytotoxicity of dLhCG and C/B-dLhCG was also evaluated as controls.

\section{Results}

\subsection{Characterization of CSD and BCD}

From the DLS results, the size of BCD (Fig. 2b) was mainly distributed at around $8 \mathrm{~nm}$ with a slight increase compared to that of BSA (Fig. 2a). The size of CSD (Fig. 2d) was mostly distributed at around $75 \mathrm{~nm}$ with an obvious increase compared to that of CS (Fig. 2c) which was less than $2 \mathrm{~nm}$ in size. After coating onto the mica surface, BCD and CSD showed nanostructured surface morphologies with the topology of several nanometers (Fig. 3).

CS was modified with dopamine via an EDC/NHS coupling (Fig. S1a $\uparrow$ ). The appearance of a peak at around $6.7 \mathrm{ppm}$ in the ${ }^{1} \mathrm{H}$ NMR spectrum of CSD which belongs to $\mathrm{H}$ on the benzene ring of dopamine confirmed the successful addition of dopamine to CS (Fig. S1 $\mathrm{b}_{\dagger}$ ). Moreover, the two peaks at around $2.8 \mathrm{ppm}$ and $2.3 \mathrm{ppm}$ in the ${ }^{1} \mathrm{H}$ NMR spectrum of CSD belong to $-\mathrm{CH}_{2}$ - of dopamine, further proving successful synthesis of CSD. The synthesis of BCD has been discussed in our previous studies. ${ }^{33,49}$

ATR-FTIR spectroscopy was also carried out for the determination of the chemical structures of CS, CSD, BSA and BCD. The appearance of a peak at around $1549 \mathrm{~cm}^{-1}$ in the ATR-FTIR spectrum of CSD confirmed the existence of the benzene ring belonging to dopamine on CSD (Fig. S2a †). 

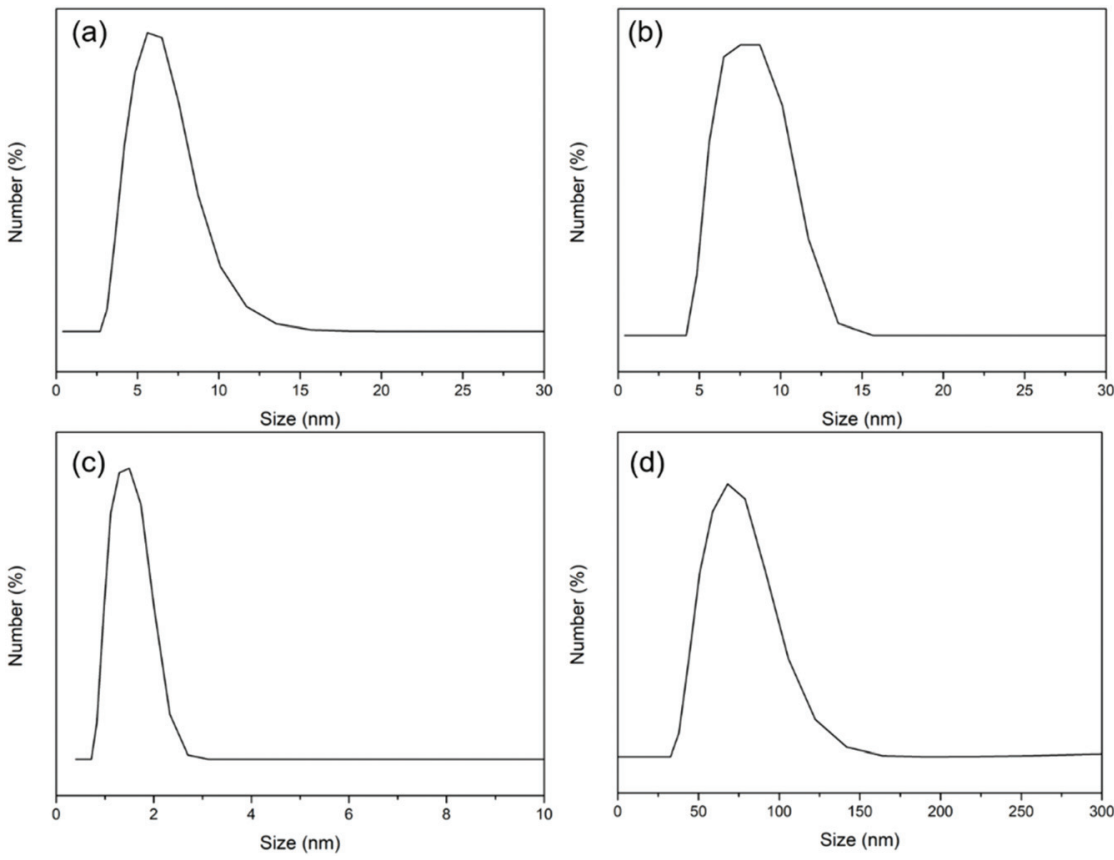

Fig. 2 The DLS results of (a) BSA, (b) BCD, (c) CS and (d) CSD, respectively.

However, there was no obvious difference in the ATR-FTIR spectrum between BSA and BCD since BSA is a protein with the benzene ring from units like tyrosine (Tyr) and phenylalanine (Phe). The peak at around $1648 \mathrm{~cm}^{-1}$ in the ATR-FTIR spectra of BSA and BCD belongs to amide I of the protein, while those at around $1285 \mathrm{~cm}^{-1}$ and $1393 \mathrm{~cm}^{-1}$ belong to amide III (Fig. S2b†).

In order to evaluate the feasibility of CSD or BCD to form a nano-coating on dLhCG, cartilage was used as the substrate for wettability measurement. dLhCG was derived from chondrocytes, composed mainly of chondrocyte secreted ECM which is rich in type II collagen and designed to reconstruct the cartilage structure. Therefore, cartilage herein could mimic dLhCG for the water contact angle test. The decrease of the water contact angle of the cartilage surface from $77.9^{\circ} \pm 2.2^{\circ}$ to $32.8^{\circ} \pm 3.5^{\circ}$ after coating CSD confirmed the existence and successful adhesion of CSD on cartilage (Fig. 4). Similarly, the decrease of the water contact angle of the cartilage surface from $77.9^{\circ} \pm 2.2^{\circ}$ to $60.2^{\circ} \pm 5.8^{\circ}$ after coating BCD confirmed the existence and successful adhesion of $\mathrm{BCD}$ on cartilage (Fig. 4).

\subsection{Morphology of grafts}

Chondrocytes' migration into grafts after implantation is of vital importance for cartilage repair and regeneration. Therefore, whether the porous structure of dLhCG would be blocked or not after coating with CSD or BCD needs to be investigated. Furthermore, both C/B-dLhCG-wash and C/B-dLhCG-no wash were observed under SEM.

As is shown in the SEM images, dLhCG had a porous structure with a pore size of hundreds of microns. CSD macromolecules also had a porous structure (Fig. 5a and e). After coating with CSD, dLhCG remained porous with decreased pore sizes (around tens of microns). In particular, a honeycomb-like structure was observed on the surface of C-dLhCGno wash with a pore size of around tens of microns.

BCD showed a sheet-like structure under SEM (Fig. 6a and e). BCD was observed twined on dLhCG for B-dLhCG-wash (Fig. 6c and g) and accumulated on the surface of dLhCG in the form of sheets for B-dLhCG-no wash (Fig. 6d and h). It is worth noting that B-dLhCG-wash changed the least in pore size in comparison with dLhCG.

TISSEEL, a widely used commercially available fibrin glue, was also applied on dLhCG and observed under SEM. TISSEEL consists of two main components, one of which is human fibrinogen, a clottable protein, and the other of which is human thrombin and it could clot and seal in seconds. Although TISSEEL is nowadays a dominant sealant in market with advantages of immediate and strong adhesion between tissues for wound closing, its resultant coagulating clot without a porous structure might block graft pores when applied in tissue engineering for fixation of grafts, which leads to failure in cell migration and finally failure of grafts and makes it insufficient for tissue engineering. As is shown in the SEM images in Fig. S $3, \dagger$ the porous structure of dLhCG after covered with TISSEEL was blocked.

\subsection{Lap shear strength}

In order to quantify the interfacing affinity between grafts and tissues and further evaluate the effect of the adhesive nanocoatings, lap shear strength test was conducted. The adhesion between grafts and cartilage was measured by lap shear test, before which factors including the CSD or BCD concentration, $\mathrm{Fe}^{3+}$ concentration and incubation time at $37^{\circ} \mathrm{C}$ in a water 

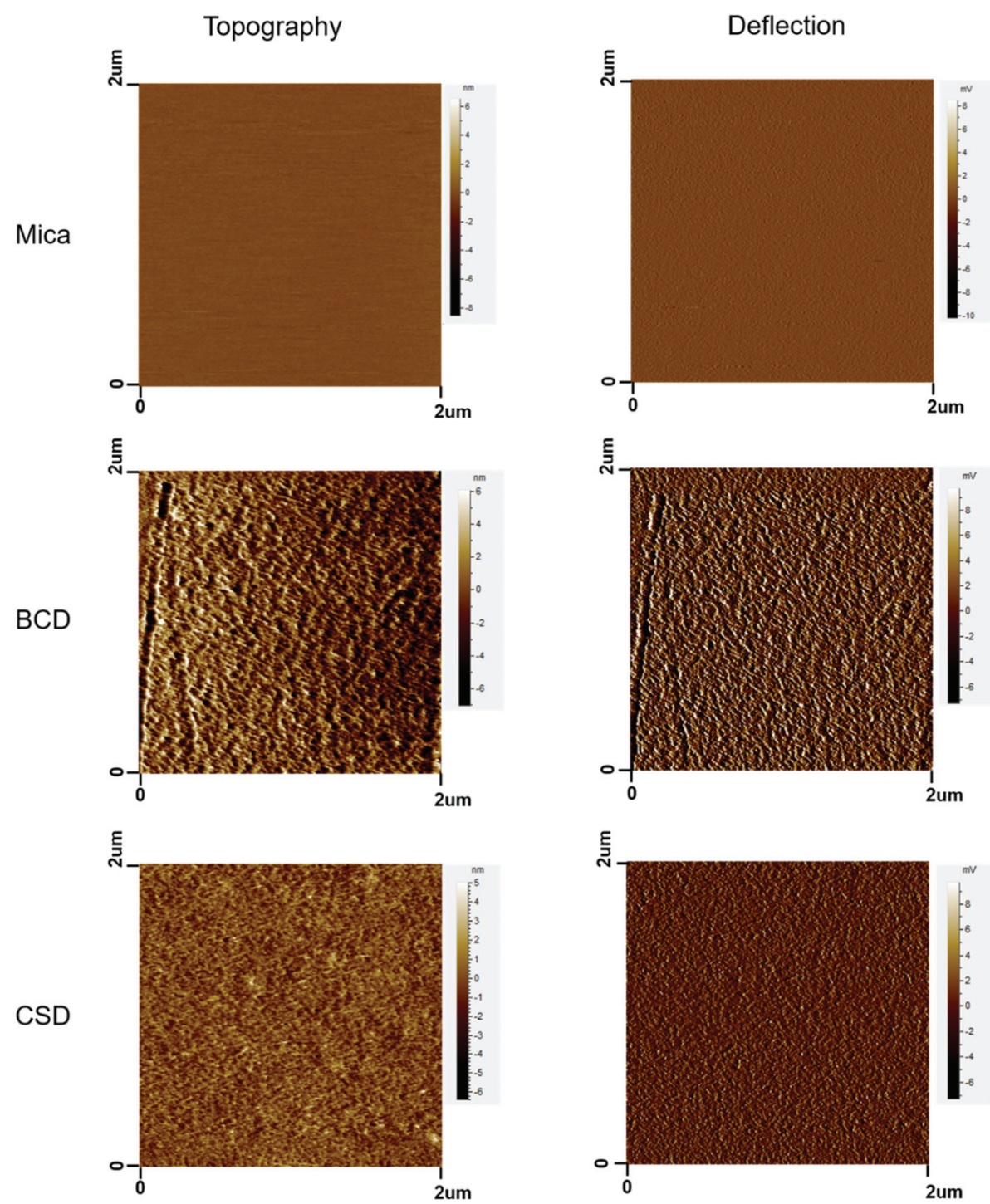

Fig. 3 AFM images of mica, BCD coated mica and CSD coated mica.

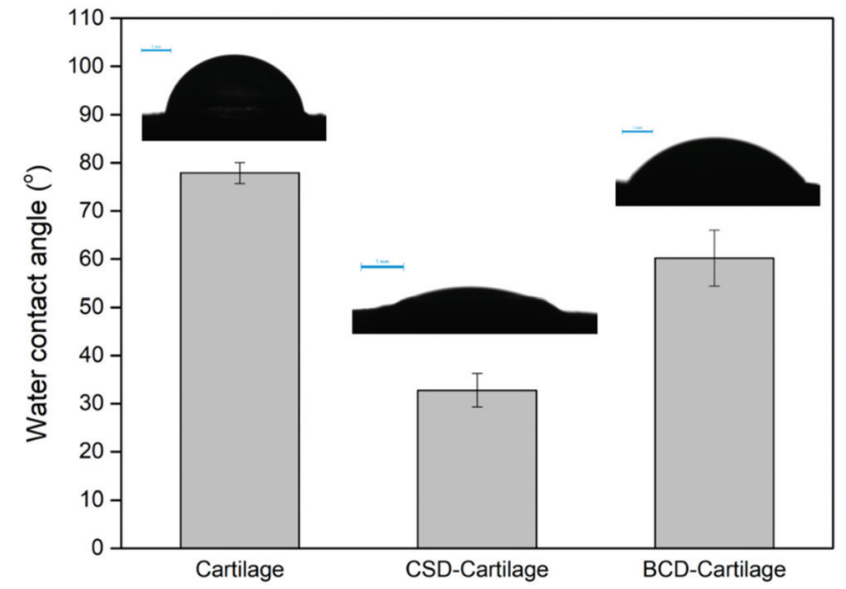

Fig. 4 Water contact angle of the cartilage surface and CSD or BCD coated cartilage surfaces. bath were optimized by lap shear test between two slices of cartilage.

For determination of the CSD or BCD concentration, the $\mathrm{Fe}^{3+}$ concentration and incubation time were fixed at $50 \mathrm{mM}$ and $3 \mathrm{~h}$, respectively. It is shown in Fig. S4a $\uparrow$ that there was a decrease in the lap shear strength when the BCD concentration was decreased

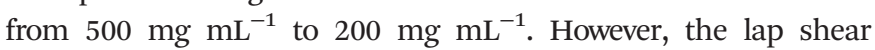
strength increased to $69.9 \pm 11.1 \mathrm{kPa}$ and $78.3 \pm 10.0 \mathrm{kPa}$ after the BCD concentration was further decreased to $100 \mathrm{mg} \mathrm{mL}^{-1}$ and $50 \mathrm{mg} \mathrm{mL}^{-1}$, respectively. The highest lap shear strength $(163.3 \pm$ $9.1 \mathrm{kPa}$ ) was achieved when the CSD concentration reached $200 \mathrm{mg} \mathrm{mL}{ }^{-1}$. CSD of the higher concentration $\left(500 \mathrm{mg} \mathrm{mL}^{-1}\right)$ and lower concentration $\left(100 \mathrm{mg} \mathrm{mL}^{-1}\right.$ and $50 \mathrm{mg} \mathrm{mL}^{-1}$ ) resulted in a much lower lap shear strength with $38.4 \pm 3.9 \mathrm{kPa}$ at $500 \mathrm{mg}$ $\mathrm{mL}^{-1}$ and $9.4 \pm 0.3 \mathrm{kPa}, 8.3 \pm 0.3 \mathrm{kPa}$ at $100 \mathrm{mg} \mathrm{mL}^{-1}$ and $50 \mathrm{mg}$ $\mathrm{mL}^{-1}$, respectively (Fig. S4d $\dagger$ ). Therefore, $50 \mathrm{mg} \mathrm{mL}^{-1} \mathrm{BCD}$ and $200 \mathrm{mg} \mathrm{mL}^{-1} \mathrm{CSD}$ were used for further optimization. 

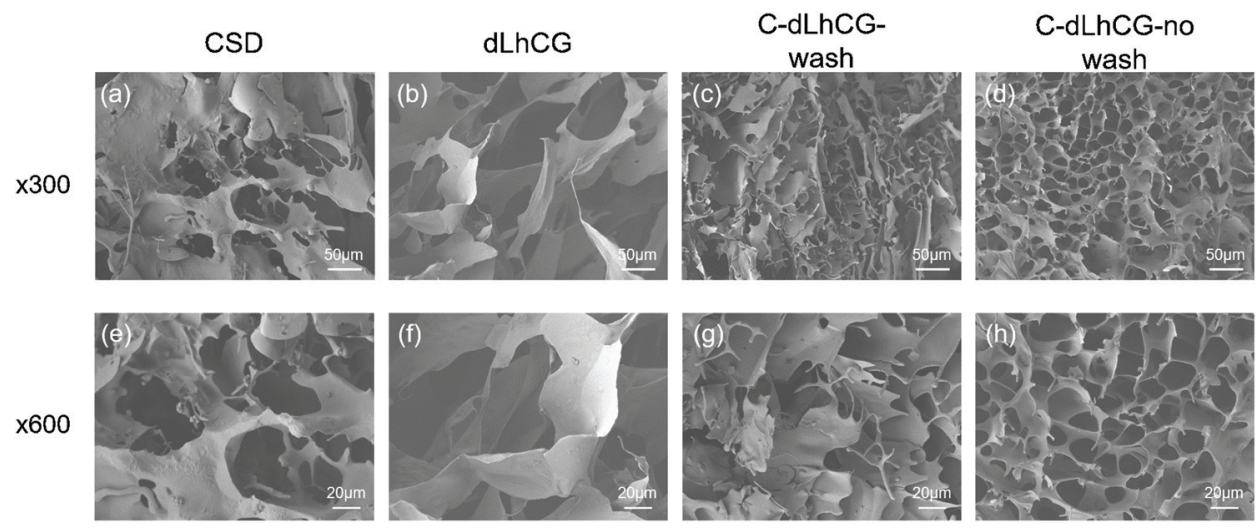

Fig. 5 SEM images of ( $a$ and e) CSD, (b and f) dLhCG, (c and g) C-dLhCG-wash and (d and h) C-dLhCG-no wash under ×300, x600 magnificent, respectively.
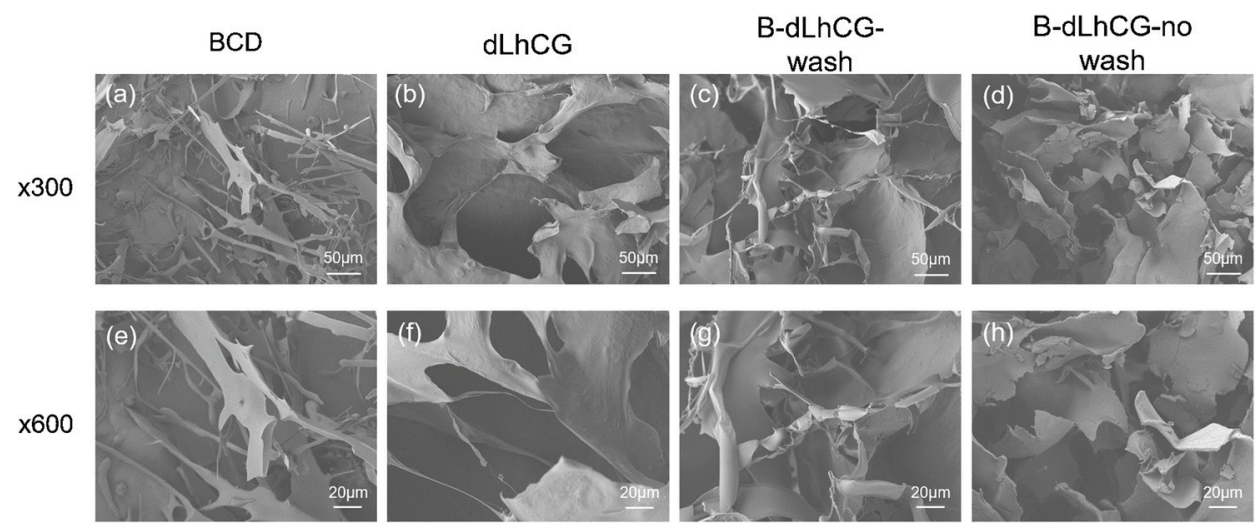

Fig. 6 SEM images of (a and e) BCD, (b and f) dLhCG, (c and g) B-dLhCG-wash and (d and h) B-dLhCG-no wash under $\times 300, \times 600$ magnificent, respectively.

For determination of the $\mathrm{Fe}^{3+}$ concentration, $50 \mathrm{mg} \mathrm{mL} \mathrm{L}^{-1}$ BCD and $200 \mathrm{mg} \mathrm{mL}^{-1} \mathrm{CSD}$ were used with incubation time fixed at $3 \mathrm{~h}$. For $50 \mathrm{mg} \mathrm{mL}^{-1} \mathrm{BCD}$, there was an increase in the lap shear strength when $\mathrm{Fe}^{3+}$ concentration was decreased from $200 \mathrm{mM}$ to $100 \mathrm{mM}$, after which a downward trend was observed as the $\mathrm{Fe}^{3+}$ concentration further decreased from $100 \mathrm{mM}$ to 0 , indicating $100 \mathrm{mM}$ as the optimized $\mathrm{Fe}^{3+}$ concentration with a lap shear strength of $136.2 \pm 14.8 \mathrm{kPa}$ (Fig. S4b $\dagger$ ). As is shown in Fig. S4e, $\uparrow$ the lap shear strength increased as the $\mathrm{Fe}^{3+}$ concentration decreased from $200 \mathrm{mM}$ to $50 \mathrm{mM}$ with a peak value of $163.3 \pm 9.1 \mathrm{kPa}$ at $50 \mathrm{mM}$. Then CSD lost adhesion after the $\mathrm{Fe}^{3+}$ concentration reached as low as $10 \mathrm{mM}$. Moreover, $50 \mathrm{mg} \mathrm{mL}{ }^{-1} \mathrm{BCD}$ by itself without chelating showed weak adhesion between cartilages $(3.7 \pm 1.8 \mathrm{kPa})$, while $200 \mathrm{mg} \mathrm{mL}^{-1} \mathrm{CSD}$ without chelating did not show potential as adhesive by itself.

Therefore, $200 \mathrm{mg} \mathrm{mL}^{-1} \mathrm{CSD}$ chelated with $50 \mathrm{mM} \mathrm{FeCl}_{3}$ and $50 \mathrm{mg} \mathrm{mL}{ }^{-1} \mathrm{BCD}$ chelated with $100 \mathrm{mM} \mathrm{FeCl}_{3}$ were chosen for optimization of the incubation time. Both showed the strongest adhesion after $3 \mathrm{~h}$ incubation at $37^{\circ} \mathrm{C}$ in a water bath (Fig. S4c and S4f as the incubation time prolonged for BCD. For CSD, little difference was observed in the lap shear strength after 15 minor $1 \mathrm{~h}$ incubation.

As a result, $200 \mathrm{mg} \mathrm{mL}^{-1} \mathrm{CSD}$ chelated with $50 \mathrm{mM} \mathrm{FeCl}_{3}$ and $50 \mathrm{mg} \mathrm{mL}^{-1}$ BCD chelated with $100 \mathrm{mM} \mathrm{FeCl}_{3}$ with an incubation time of $3 \mathrm{~h}$ were chosen as the optimized working conditions for adhesion.

With optimal working concentrations and incubation time determined, the lap shear strength test between grafts and cartilage was conducted based on the model shown in Fig. 7a. The lap shear strengths of dLhCG swelling solely in Tris-HCl buffer or $\mathrm{FeCl}_{3}$ solution of certain concentration $(50 \mathrm{mM}$ for CSD related groups and $100 \mathrm{mM}$ for BCD related groups) and C/B-dLhCG were also measured as controls. Both CFe-dLhCG and BFe-dLhCG showed an enhanced lap shear strength with cartilage compared to dLhCG (Fig. 7b and c). In particular, the lap shear strength between CFe-dLhCG-wash and cartilage reached $72.6 \pm 8.3 \mathrm{kPa}$, while that of CFe-dLhCG-no wash with cartilage was $70.9 \pm 19.6 \mathrm{kPa}$ (Fig. 7b). Similarly, the lap shear strength between BFe-dLhCG-wash and cartilage reached 55.2 $\pm 0.8 \mathrm{kPa}$, while that of BFe-dLhCG-no wash with cartilage was $62.3 \pm 9.4 \mathrm{kPa}$ (Fig. 7c). Moreover, B-dLhCG exhibited a slightly higher lap shear strength with cartilage $(3.5 \pm 0.3 \mathrm{kPa})$ than 

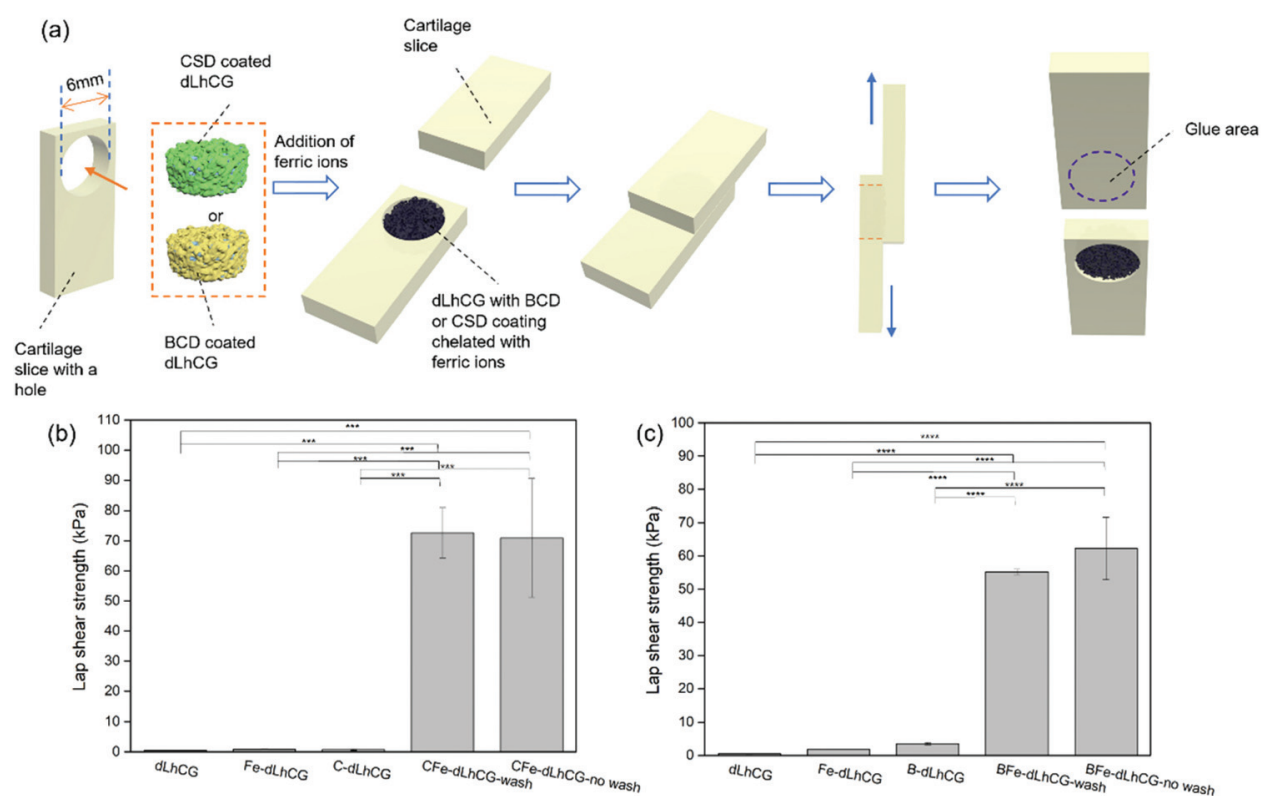

Fig. 7 (a) Schematic illustration of the lap shear strength test between cartilage and graft; the lap shear strength between cartilage and (b) dLhCG, Fe-dLhCG, C-dLhCG, CFe-dLhCG-wash and CFe-dLhCG-no wash or (c) dLhCG, Fe-dLhCG, B-dLhCG, BFe-dLhCG-wash and BFe-dLhCG-no wash, respectively $\left({ }^{*} p<0.05,{ }^{* *} p<0.01,{ }^{* * *} p<0.001\right.$ and $\left.{ }^{* * * *} p<0.0001\right)$.

dLhCG $(0.5 \pm 0.1 \mathrm{kPa})$, further confirming BCD's adhesion by nature. However, no obvious difference was found between the samples that were washed and not washed before freezedrying during fabrication, which indicates that covering dLhCG with accumulated CSD or BCD does not make any difference compared to coating dLhCG with CSD or BCD through adherence by dopamine. What's worse, the accumulated CSD or BCD on dLhCG would result in a reduced pore size smaller than the washed samples according to SEM images (Fig. 5 and 6). Therefore, CFe/BFe-dLhCG-wash was selected for further investigations on the cells. C/B-dLhCG and CFe/BFe-dLhCG in the following Section 3.4 and Discussion refer in particular to C/B-dLhCG-wash and CFe/ BFe-dLhCG-wash, respectively.

\subsection{In vitro cytotoxicity}

In order to figure out if the CSD or BCD and ferric ions have influence on chondrocyte viability and further the possibility of medical use, in vitro cytotoxicity was measured by WST-1 based on the $\mathrm{Fe}^{3+}$ concentration and $\mathrm{CSD} / \mathrm{BCD}$ concentration. First of all, chondrocytes incubated together with dLhCG showed viabilities of $103.0 \pm 6.3 \%, 106.1 \pm 24.4 \%$ and $106.2 \pm$ $10.4 \%$ on day 1 , day 4 and day 7 , respectively. The effect of $\mathrm{Fe}^{3+}$ concentration on cytotoxicity involving $200 \mathrm{mM}, 50 \mathrm{mM}$ and $10 \mathrm{mM}$ was investigated and is shown in Fig. 8d-f. BCD and CSD involved herein were fixed at $200 \mathrm{mg} \mathrm{mL} \mathrm{mL}^{-1}$. According to the results, the introduction of CSD or BCD did not sacrifice much cytocompatibility of dLhCG, and showed comparable cell viability between C-dLhCG and B-dLhCG in general. However, when C/B-dLhCG chelated with $200 \mathrm{mM}$ $\mathrm{FeCl}_{3}$, chondrocyte viability remarkably reduced. Moreover, there is an increase in chondrocyte viability with the decrease of the $\mathrm{FeCl}_{3}$ concentration for BFe-dLhCG. As for CFe-dLhCG, the cytotoxicity increased as the $\mathrm{FeCl}_{3}$ concentration increased on day 1. However, there was no obvious difference between $50 \mathrm{mM} \mathrm{FeCl}_{3}$ and $10 \mathrm{mM} \mathrm{FeCl}_{3}$, which showed better cytocompatibility than $200 \mathrm{mM} \mathrm{FeCl}_{3}$ on day 4 and day 7 .

The effect of various CSD or BCD concentrations on cytotoxicity is shown in Fig. $8 \mathrm{a}-\mathrm{c}$. The $\mathrm{FeCl}_{3}$ concentration involved herein was fixed at $50 \mathrm{mM}$. For C-dLhCG and CFe-dLhCG, a rough trend of the lower CSD concentration accompanying with better cytocompatibility was observed. As for B-dLhCG and BFedLhCG, the BCD concentration did not have obvious influence on chondrocyte viability on day 1 , while a BCD concentration of $50 \mathrm{mg} \mathrm{mL}{ }^{-1}$ showed mildly better performance than those of $100 \mathrm{mg} \mathrm{mL}^{-1}$ and $200 \mathrm{mg} \mathrm{mL}^{-1}$ on day 4 and day 7 in general.

\section{Discussion}

In this study, one problem of tissue engineering to be addressed, the interfacing affinity of grafts on host tissues which referred in particular to cartilage herein was paid attention to and investigated. Two bio-adhesive nano-coatings developed by our group, CSD and BCD, were introduced in this study and coated onto dLhCG to enhance the adhesion between cartilage and dLhCG, a tissue-engineered cartilage graft developed by our group. Adhesion and cohesion were achieved after the addition of $\mathrm{FeCl}_{3}$ and by the chelation between catechol on dopamine and $\mathrm{Fe}^{3+}$ ions. By fabricating bio-adhesive nano-coatings and further adhesive grafts, the integration of grafts with surrounding tissues in the defects can be achieved in situ by the addition of ferric ions instead of preparing and applying bio-adhesives separately post-implan- 

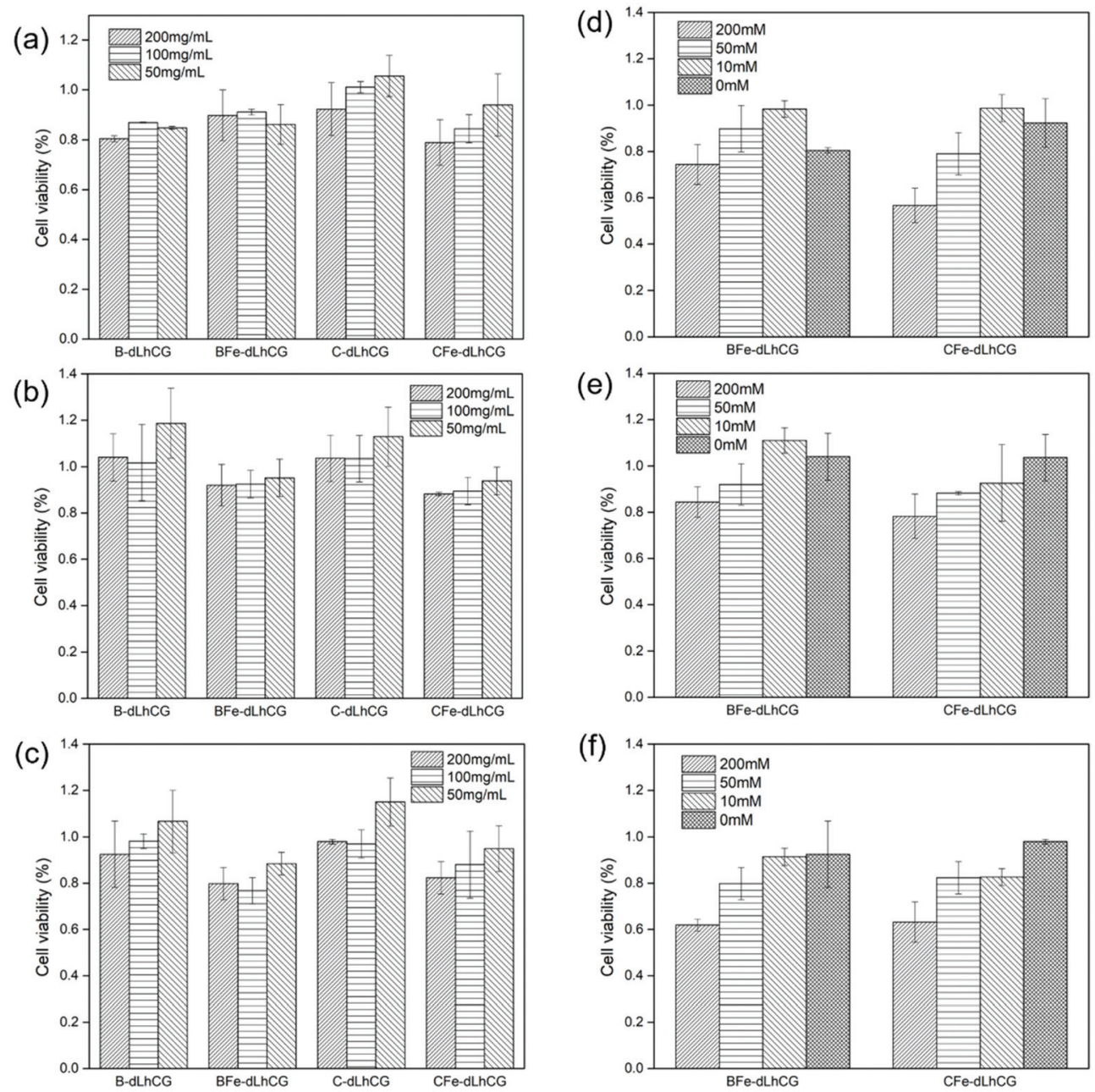

Fig. 8 WST-1 results based on different CSD or BCD concentration on (a) day 1, (b) day 4, (c) day 7 and WST-1 results based on different Fe ${ }^{3+}$ concentration on (d) day 1 , (e) day 4, (f) day 7.

tation. CS, a cartilage derived sulfate GAG, or BSA, a common biocompatible plasma protein, were selected as the backbone for the adhesives, respectively. Dopamine, a mussel-inspired adhesive molecule, was introduced as a functional unit to carry out adhesive studies by chelating with $\mathrm{Fe}^{3+}$ in an alkaline environment. CS is an ideal backbone of adhesive aimed at cartilage tissue engineering since CS is cartilage-derived, which indicates that no impurities will be introduced when CS is used. BCD was also investigated considering its good performance as a sealant. ${ }^{33}$ Dopamine can adhere to various surfaces through oxidation/polymerization of catechol groups, which contributed to the adhesion of BCD or CSD on dLhCG and cartilage surfaces. In addition, the cohesion of BCD or CSD was based on chelation between catechol groups and ferric ions.

The oxidation/polymerization of catechol groups involves both chemical and physical interactions, while the chelation with ferric ions belongs to chemical interactions. It makes the nano-coatings irreversible and stable both physically and chemically due to the robust chemical bonds. The bio-adhesive nano-coatings are bioabsorbable and can be digested by the host in vivo. However, after several weeks post-implantation, the cells will migrate, proliferate and develop in the regenerated sites because of the favorable microenvironment created by the ECM-based tissue-engineered grafts. Therefore, the grafts will integrate with surrounding tissues by growth and the ECM secreted by migrated cells several weeks post-implantation, and the main target of the bio-adhesive nano-coatings is to ensure the integration of grafts with surrounding tissues in the early stage of tissue regeneration post-implantation.

First of all, CSD and BCD, whose chemical structures were characterized by ${ }^{1} \mathrm{H}$ NMR and ATR-FTIR spectroscopy, were synthesized by EDC/NHS coupling. The appearance of a peak at around $6.7 \mathrm{ppm}$ in the ${ }^{1} \mathrm{H}$ NMR spectrum of CSD (Fig. S1b $\dagger$ ) and the peak at around $1549 \mathrm{~cm}^{-1}$ in the ATR-FTIR spectrum of CSD (Fig. S2a $\dagger$ ) confirmed the existence of the benzene ring belonging to dopamine on CSD. Moreover, the two peaks at around $2.8 \mathrm{ppm}$ and $2.3 \mathrm{ppm}$ in the ${ }^{1} \mathrm{H}$ NMR spectrum of CSD belonging to $-\mathrm{CH}_{2}$ - of dopamine also proved the successful synthesis of CSD. The chemical structures of BCD could be referred to in our previous studies. ${ }^{33,49}$ BCD and CSD were 
around $8 \mathrm{~nm}$ and $75 \mathrm{~nm}$ in size, respectively, according to the DLS results (Fig. 2) and demonstrated nanostructures as surface coatings based on AFM images (Fig. 3).

CSD and BCD were designed to coat on dLhCG first by the polymerization of dopamine in an alkaline environment. Before coating CSD or BCD on dLhCG, the feasibility of CSD or BCD to retain on the surface of dLhCG as a nano-coating was investigated with the cartilage surface as a mimic of the dLhCG surface. dLhCG, a chondrocyte-derived cartilage graft composed mainly of the chondrocyte secreted ECM, was developed as a graft to restore the cartilage components and structure for cartilage tissue repair. However, its porous structure made it difficult for surface analysis such as wettability test. Therefore, the porcine cartilage surface, which shares similar source and structure with dLhCG but is flatter, was chosen as a mimic of the dLhCG surface for water contact angle test. The decrease of the water contact angle of the cartilage surface from $77.9^{\circ} \pm 2.2^{\circ}$ to $32.8^{\circ} \pm 3.5^{\circ}$ and $60.2^{\circ} \pm 5.8^{\circ}$ after coating CSD and BCD, respectively, confirmed the existence and successful adhesion of CSD and BCD on cartilage (Fig. 4). Furthermore, it is also demonstrated that the adhesion of BCD or CSD to dLhCG and the cartilage surfaces was achieved through oxidation/polymerization of catechol groups.

Whether CSD or BCD coating will block the porous structures of dLhCG or not is the first issue that should be taken into consideration. After determining the structures of CSD and BCD and their feasibility to form coatings on dLhCG, we carried out SEM observation for morphology characterization. It is observed that CSD had a porous structure while BCD had a sheet structure. The pore size of dLhCG decreased to tens of microns from hundreds of microns with CSD coating, especially C-dLhCG-no wash. C-dLhCG-no wash was observed with a honeycomb-like structure (Fig. 5d and h). BCD was detected twining on dLhCG from the SEM images of B-dLhCGwash (Fig. 6c and g) and accumulated on dLhCG sheets by sheets from the SEM images of B-dLhCG-no wash (Fig. 6d and h). A decrease in the pore size of B-dLhCG-wash and B-dLhCGno wash was also observed but not as obvious as those of C-dLhCG-wash and C-dLhCG-no wash.

The adhesive strength between grafts and cartilage was investigated in the form of the lap shear strength, before which the working concentration of CSD or BCD and $\mathrm{Fe}^{3+}$ and the incubation time at $37{ }^{\circ} \mathrm{C}$ in a water bath were optimized. An optimization was necessary in that adhesion was mainly achieved by chelating between $\mathrm{Fe}^{3+}$ with catechol groups on dopamine, and the chelation structure was reported to be one ferric ion bond with three catechol groups. ${ }^{50}$ An optimal combination of the CSD or BCD concentration and $\mathrm{Fe}^{3+}$ concentration should be found. For the BCD concentration, adhesion became generally stronger as the BCD concentration decreased from $500 \mathrm{mg} \mathrm{mL}^{-1}$ to $50 \mathrm{mg} \mathrm{mL}^{-1}$ (Fig. S4a†), thus $50 \mathrm{mg}$ $\mathrm{mL}^{-1} \mathrm{BCD}$ with a highest lap shear strength of $78.3 \pm 10.0 \mathrm{kPa}$ was chosen as the working concentration, after which a $\mathrm{Fe}^{3+}$ concentration of $100 \mathrm{mM}$ was chosen (Fig. S4b†). For the CSD concentration, the strongest adhesion (163.3 $\pm 9.1 \mathrm{kPa})$ appeared when $200 \mathrm{mg} \mathrm{mL}^{-1} \mathrm{CSD}$ was applied (Fig. S4d $\dagger$ ) with a $\mathrm{Fe}^{3+}$ concentration of $50 \mathrm{mM}$ (Fig. S4 $\mathrm{e}_{\dagger}$ ). Moreover, the adhesive strength was enhanced with an increased incubation time (Fig. S4c and S4f $\dagger$ ). Finally, an incubation time of $3 \mathrm{~h}$ was chosen.

The optimized working concentration and incubation time were applied further in the measurement of the adhesive strength between dLhCG and cartilage. dLhCG with both CSD coating and $\mathrm{Fe}^{3+}$ chelating showed an enhanced adhesive strength of over $70 \mathrm{kPa}$ with CFe-dLhCG-wash of $72.6 \pm 8.3 \mathrm{kPa}$ and CFe-dLhCG-no wash of $70.9 \pm 19.6 \mathrm{kPa}$. Similarly, both BFe-dLhCG-wash (55.2 $\pm 0.8 \mathrm{kPa})$ and BFe-dLhCG-no wash $(62.3 \pm 9.4 \mathrm{kPa})$ also showed enhanced adhesive strength. However, no obvious difference was found between the two kinds of process methods (wash and no wash), indicating that covering dLhCG with accumulated CSD or BCD by an external force would not contribute to better adhesive performances. It might be due to the failure of contact and function of excess CSD or BCD at the interfaces as an effective component. Therefore, CFe-dLhCG-wash and BFe-dLhCG-wash were chosen for further investigations with cells, and CFe/BFe-dLhCG shown in Fig. 8 and further discussion involving in vitro cytotoxicity referred in particular to $\mathrm{CFe} / \mathrm{BFe}-\mathrm{dLhCG}-w a s h$. Moreover, it is worth noting that no obvious enhancement in the adhesion strength was found by coating BCD or CSD only, indicating that the cohesion was mainly based on chelation between the catechol groups and ferric ions.

In vitro cytotoxicity of chondrocytes was studied to find out the influences of the CSD or BCD concentration and $\mathrm{Fe}^{3+}$ concentration on chondrocyte viability. As shown in Fig. 8, higher cell viability was observed with a lower $\mathrm{FeCl}_{3}$ concentration from $200 \mathrm{mM}$ to $10 \mathrm{mM}$ in BFe-dLhCG. B-dLhCG showed relatively higher cytotoxicity than BFe-dLhCG with $10 \mathrm{mM} \mathrm{Fe}^{3+}$ on day 1 and day 4, which might be caused by the release of BCD solution to the medium in the first few days considering the small sheet-like structure of BCD (Fig. 6a and e). There was generally an increase in chondrocyte viability with a decreased $\mathrm{FeCl}_{3}$ concentration from $200 \mathrm{mM}$ to 0 in CFe-dLhCG. The $50 \mathrm{mM} \mathrm{FeCl}_{3}$ concentration samples and $10 \mathrm{mM} \mathrm{FeCl}_{3}$ concentration samples showed similar cell viability on day 4 and day 7 for CFe-dLhCG. As is shown in Fig. 8, chondrocyte viability was slightly weakened by CSD of a higher concentration but was nearly not affected by the BCD concentration in general. dLhCG was derived from chondrocytes and created a favorable microenvironment for cells. Therefore, according to the results for $\mathrm{CFe} / \mathrm{BFe}-\mathrm{dLhCG}$, the ferric ion's concentration plays a relatively more significant role in cytotoxicity compared to the CSD or BCD concentration.

In this study, the dLhCG graft coated with adhesive nanocoatings CSD and BCD retained significantly enhanced adhesion with surrounding cartilage tissues in a highly humid environment (via incubation at $37^{\circ} \mathrm{C}$ in a water bath) without immersion in water phase; nevertheless, to keep the adhesive strength in water phase remains an unsolved challenge for hydrophilic tissue adhesives due to the effect of water swelling, which has drawn extensive attention of researchers over decades. ${ }^{51-60}$ Nowadays, the introduction of hydrophobicity in 
adhesive gels or fibers has been emphasized for underwater adhesion. ${ }^{61-65}$ Accordingly, we propose to introduce hydrophobic components in the design of future research as a waterproof agent for the adhesive nano-coating to maintain the high adhesion in an underwater environment.

\section{Conclusion}

In this study, CSD and BCD were coated onto dLhCG as adhesive nano-coatings, respectively, for further fixation with cartilage by the addition of ferric ions. CS, a cartilage derived sulfated GAG, was chosen as the main backbone, which would introduce no impurities for cartilage tissue engineering. BSA, a universal biocompatible plasma protein, was also used as the main backbone. Dopamine was introduced on CS and BSA, the catechol groups on which can polymerize with existence of oxygen and chelate with ferric ions in an alkaline environment. CSD and BCD were attached to dLhCG first mainly via the self-polymerization of dopamine. Then CSD or BCD coated dLhCG was fixed in defects of cartilage by the addition of Tris-HCl buffer $(10 \mathrm{mM}$, $\mathrm{pH}$ 8.5) and ferric ions to form an interpenetrating network for adhesion. Both CFe-dLhCG (up to $70 \mathrm{kPa}$ ) and BFe-dLhCG (up to $55 \mathrm{kPa}$ ) showed enhanced adhesive strength with cartilage compared to dLhCG. In summary, CSD is superior as an adhesive nano-coating for graft fixation in retaining porous structures, introducing no impurities, enhancing adhesion between grafts and surrounding tissues. BCD is another suitable adhesive nano-coating for integrating grafts with surrounding tissues in porous structures and favoring biocompatibility.

\section{Conflicts of interest}

There are no conflicts to declare.

\section{Acknowledgements}

This work was supported by the Shenzhen-Hong Kong Innovation Circle Category D Project (SGDX2019081623180779 to Dong-An Wang), Science, Technology and Innovation Commission of Shenzhen Municipality, China; the National Natural Science Foundation of China (NSFC51973180); the General Research Fund, Research Grants Council, University Grants Committee, Hong Kong SAR (CityU 11205520); the grant from Karolinska Institutet Ming Wai Lau Centre of Reparative Medicine (to Dong-An Wang); and the grants from City University of Hong Kong (SGP 9380099, 9231412, 9680269, 9678192, and 7005212).

\section{References}

1 Y. Gao, X. Han, J. Chen, Y. Pan, M. Yang, L. Lu, J. Yang, Z. Suo and T. Lu, Proc. Natl. Acad. Sci. U. S. A., 2021, 118, e2103457118.
2 H. H. Lu, S. D. Subramony, M. K. Boushell and X. Zhang, Ann. Biomed. Eng., 2010, 38, 2142-2154.

3 J. F. Kukleta, C. Freytag and M. Weber, Hernia, 2012, 16, 153-162.

4 J. C. Reichert, S. Saifzadeh, M. E. Wullschleger, D. R. Epari, M. A. Schütz, G. N. Duda, H. Schell, M. van Griensven, H. Redl and D. W. Hutmacher, Biomaterials, 2009, 30, 2149-2163.

5 Y. Gong, K. Su, T. T. Lau, R. Zhou and D. A. Wang, Tissue Eng., Part A, 2010, 16, 3611-3622.

6 K. Su, T. T. Lau, W. Leong, Y. Gong and D. A. Wang, Adv. Funct. Mater., 2012, 22, 972-978.

7 W. Leong, T. T. Lau and D. A. Wang, Acta Biomater., 2013, 9, 6459-6467.

8 Y. Peck, P. He, G. S. Chilla, C. L. Poh and D. A. Wang, Sci. Rep., 2015, 5, 16225.

9 X. Nie and D. Wang, Biomater. Sci., 2018, 6, 2798-2811.

10 X. Nie, J. Yang, Y. J. Chuah, W. Zhu, Y. Peck, P. He and D. Wang, Adv. Healthcare Mater., 2020, 9, 1901304.

11 X. Nie, Y. J. Chuah, W. Zhu, P. He, Y. Peck and D. A. Wang, Biomaterials, 2020, 235, 119821.

12 A. Kassam, M. Horowitz, R. Carrau, C. Snyderman, W. Welch, B. Hirsch and Y. F. Chang, Neurosurgery, 2003, 52, 1102-1105.

13 V. Holmdahl, O. Backman, U. Gunnarsson and K. Strigård, Front. Surg., 2019, 6, 2-7.

14 A. R. Brackeen, M. J. Wells and J. M. Freed, Dermatol. Surg., 2005, 31, 1707-1709.

15 X. Meng, X. Wang, Y. Jiang, B. Zhang, K. Li and Q. Li, RSC Adv., 2019, 9, 21258-21264.

16 M. Marni and A. Ferrero, Transplantation, 1987, 575-577.

17 J. McCue and L. K. Kalliainen, Ann. Surg. Oncol., 2018, 25, 471-474.

18 D. B. Horváthy, G. Vácz, A. Cselenyák, M. Weszl, L. Kiss and Z. Lacza, Surg. Innov., 2013, 20, 249-255.

19 J. Tipson, Plast. Reconstr. Surg., 1989, 83, 194-195.

20 P. J. M. Bouten, M. Zonjee, J. Bender, S. T. K. Yauw, H. van Goor, J. C. M. van Hest and R. Hoogenboom, Prog. Polym. Sci., 2014, 39, 1375-1405.

21 C. Ghobril and M. W. Grinstaff, Chem. Soc. Rev., 2015, 44, 1820-1835.

22 A. Vahdati and D. R. Wagner, J. Biomech., 2013, 46, 1554-1560.

23 W. Zhu, Y. J. Chuah and D. A. Wang, Acta Biomater., 2018, 74, 1-16.

24 M. Singh, H. S. Nanda, R. D. O'Rorke, A. E. Jakus, A. H. Shah, R. N. Shah, R. D. Webster and T. W. J. Steele, Adv. Healthcare Mater., 2018, 7, 1-13.

25 C. L. Mullens, C. A. Messa, G. M. Kozak, I. A. Rhemtulla and J. P. Fischer, Plast. Reconstr. Surg. Glob. Open, 2019, 7, e2187.

26 M. Ivarsson, M. Prenkert, A. Cheema, P. Wretenberg and N. Andjelkov, Cartilage, 2019, 1-9.

27 H. Yan, L. Li, Z. Wang, Y. Wang, M. Guo, X. Shi, J. M. Yeh and P. Zhang, ACS Biomater. Sci. Eng., 2019, 6, 634-646.

28 S. Boyadzhieva, K. Sorg, M. Danner, S. C. L. Fischer, R. Hensel, B. Schick, G. Wenzel, E. Arzt and K. Kruttwig, Polymers, 2019, 11, 942. 
29 J. Sun, J. Su, C. Ma, R. Göstl, A. Herrmann, K. Liu and H. Zhang, Adv. Mater., 2020, 32, 1906360.

30 S. Bai, X. Zhang, X. Lv, M. Zhang, X. Huang, Y. Shi, C. Lu, J. Song and H. Yang, Adv. Funct. Mater., 2020, 30, $1-12$.

31 N. Annabi, Y.-N. Zhang, A. Assmann, E. S. Sani, G. Cheng, A. D. Lassaletta, A. Vegh, B. Dehghani, G. U. Ruiz-Esparza, X. Wang, S. Gangadharan, A. S. Weiss and A. Khademhosseini, Sci. Transl. Med., 2017, 9, 1-14.

32 N. Annabi, S. R. Shin, A. Tamayol, M. Miscuglio, M. A. Bakooshli, A. Assmann, P. Mostafalu, J. Y. Sun, S. Mithieux, L. Cheung, X. Tang, A. S. Weiss and A. Khademhosseini, Adv. Mater., 2016, 28, 40-49.

33 W. Zhu, Y. Peck, J. Iqbal and D. A. Wang, Biomaterials, 2017, 147, 99-115.

34 A. Serrero, S. Trombotto, Y. Bayon, P. Gravagna, S. Montanari and L. David, Biomacromolecules, 2011, 12, 1556-1566.

35 B. Balakrishnan, D. Soman, U. Payanam, A. Laurent, D. Labarre and A. Jayakrishnan, Acta Biomater., 2017, 53, 343-354.

36 C. Strehmel, H. Perez-Hernandez, Z. Zhang, A. Löbus, A. F. Lasagni and M. C. Lensen, ACS Biomater. Sci. Eng., 2015, 1, 747-752.

37 C. R. Matos-Pérez and J. J. Wilker, Macromolecules, 2012, 45, 6634-6639.

38 Y. Li, H. Meng, Y. Liu, A. Narkar and B. P. Lee, ACS Appl. Mater. Interfaces, 2016, 8, 11980-11989.

39 M. D. Phaneuf, D. J. Dempsey, M. J. Bide, W. C. Quist and F. W. LoGerfo, Biomaterials, 2001, 22, 463-469.

40 A. J. Singer, J. V. Quinn and J. E. Hollander, Am. J. Emerg. Med., 2008, 26, 490-496.

41 G. Liu, Z. Shi, T. Kuriger, L. R. Hanton, J. Simpson, S. C. Moratti, B. H. Robinson, T. Athanasiadis, R. Valentine, P. J. Wormald and S. Robinson, Macromol. Symp., 2009, 279, 151-157.

42 A. S. Sawhney, C. P. Pathak and J. A. Hubbell, Macromolecules, 1993, 26, 581-587.

43 K. Autumn, Y. A. Liang, S. T. Hsieh, W. Zesch, W. P. Chan, T. W. Kenny, R. Fearing and R. J. Full, Nature, 2000, 405, 681-685.

44 S. Y. Yang, E. D. O’Cearbhaill, G. C. Sisk, K. M. Park, W. K. Cho, M. Villiger, B. E. Bouma, B. Pomahac and J. M. Karp, Nat. Commun., 2013, 4, 1702.

45 T. G. Barclay, H. M. Hegab, S. R. Clarke and M. GinicMarkovic, Adv. Mater. Interfaces, 2017, 4, 1-38.
46 W. Zhu, J. Iqbal and D. A. Wang, J. Mater. Chem. B, 2019, 7, 1741-1752.

47 C. Fan, J. Fu, W. Zhu and D. A. Wang, Acta Biomater., 2016, 33, 51-63.

48 W. Zhu, J. Yang, J. Iqbal, Y. Peck, C. Fan and D. A. Wang, J. Surg. Res., 2017, 215, 173-182.

49 C. Tao, W. Zhu, J. Iqbal, C. Xu and D. A. Wang, J. Mater. Chem. B, 2020, 8, 6080-6091.

50 E. Filippidi, T. R. Cristiani, C. D. Eisenbach, J. H. Waite, J. N. Israelachvili, B. K. Ahn and M. T. Valentine, Science, 2017, 358, 502-505.

51 J. H. Waite, Int. J. Adhes. Adhes., 1987, 7, 9-14.

52 J. Wu, L. Zhang, Y. Wang, Y. Long, H. Gao, X. Zhang, N. Zhao, Y. Cai and J. Xu, Langmuir, 2011, 27, 13684-13691.

53 Z. Shafiq, J. Cui, L. Pastor-Pérez, V. San Miguel, R. A. Gropeanu, C. Serrano and A. del Campo, Angew. Chem., Int. Ed., 2012, 51, 4332-4335.

54 E. W. Danner, Y. Kan, M. U. Hammer, J. N. Israelachvili and J. H. Waite, Biochemistry, 2012, 51, 6511-6518.

55 S. Kim, H. Y. Yoo, J. Huang, Y. Lee, S. Park, Y. Park, S. Jin, Y. M. Jung, H. Zeng, D. S. Hwang and Y. Jho, ACS Nano, 2017, 11, 6764-6772.

56 M. A. Gebbie, W. Wei, A. M. Schrader, T. R. Cristiani, H. A. Dobbs, M. Idso, B. F. Chmelka, J. H. Waite and J. N. Israelachvili, Nat. Chem., 2017, 9, 473-479.

57 B. K. Ahn, J. Am. Chem. Soc., 2017, 139, 10166-10171.

58 C. Cui, C. Fan, Y. Wu, M. Xiao, T. Wu, D. Zhang, X. Chen, B. Liu, Z. Xu, B. Qu and W. Liu, Adv. Mater., 2019, 31, 1905761.

59 C. Ma, J. Sun, B. Li, Y. Feng, Y. Sun, L. Xiang, B. Wu, L. Xiao, B. Liu, V. S. Petrovskii, B. Liu, J. Zhang, Z. Wang, H. Li, L. Zhang, J. Li, F. Wang, R. Göstl, I. I. Potemkin, D. Chen, H. Zeng, H. Zhang, K. Liu and A. Herrmann, Nat. Commun., 2021, 12, 3613.

60 M. Lo Presti, G. Rizzo, G. M. Farinola and F. G. Omenetto, Adv. Sci., 2021, 2004786, 2004786.

61 B. Soltannia and D. Sameoto, ACS Appl. Mater. Interfaces, 2014, 6, 21995-22003.

62 Y. Xu, Q. Liu, A. Narayanan, D. Jain, A. Dhinojwala and A. Joy, Adv. Mater. Interfaces, 2017, 4, 1700506.

63 X. Liu, Q. Zhang, L. Duan and G. Gao, ACS Appl. Mater. Interfaces, 2019, 11, 6644-6651.

64 L. Han, M. Wang, L. O. Prieto-López, X. Deng and J. Cui, Adv. Funct. Mater., 2020, 30, 1907064.

65 H. Fan, J. Wang and J. P. Gong, Adv. Funct. Mater., 2021, 31, 2009334. 